\title{
BMJ Open Implementing telehealth support to increase physical activity in girls and women with Rett syndrome-ActivRett: protocol for a waitlist randomised controlled trial
}

\author{
Jenny Downs (D) , ${ }^{1,2}$ Meir Lotan, ${ }^{3}$ Cochavit Elefant, ${ }^{4}$ Helen Leonard (D) , ${ }^{1}$ \\ Kingsley Wong, ${ }^{1}$ Nicholas Buckley, ${ }^{1,2}$ Michelle Stahlhut ${ }^{5}$
}

To cite: Downs J, Lotan M, Elefant C, et al. Implementing telehealth support to increase physical activity in girls and women with Rett syndrome-ActivRett: protocol for a waitlist randomised controlled trial. BMJ Open 2020;10:e042446. doi:10.1136/ bmjopen-2020-042446

- Prepublication history and additional materials for this paper are available online. To view these files, please visit the journal online (http://dx.doi. org/10.1136/bmjopen-2020042446).

Received 05 July 2020 Revised 18 November 2020 Accepted 27 November 2020

D) Check for updates

(c) Author(s) (or their employer(s)) 2020. Re-use permitted under CC BY-NC. No commercial re-use. See rights and permissions. Published by BMJ.

For numbered affiliations see end of article.

\section{Correspondence to} Dr Jenny Downs; Jenny.Downs@telethonkids. org.au

\section{ABSTRACT}

Introduction Individuals with Rett syndrome (RTT) experience impaired gross motor skills, limiting their capacity to engage in physical activities and participation in activities. There is limited evidence of the effectiveness of supported physical activity interventions. This study aims to evaluate the effects of a telehealth-delivered physical activity programme on physical activity, sedentary behaviour and quality of life in RTT.

Methods and analysis This is a multicentre study, conducted in Australia, Denmark and Israel. It is a randomised waitlist-controlled trial comparing an intervention to support physical activity with usual care. Participants are children and adults with RTT, recruited from the Australian Rett Syndrome Database, the Danish Center for Rett Syndrome and the Rett Syndrome Association of Israel. The intervention duration is 12 weeks, including fortnightly telephone contact to plan, monitor and develop individual activity programmes. Outcomes are measured at baseline, at 13 weeks and then at 25 weeks. The primary outcomes are sedentary behaviour assessed with an activPAL accelerometer and the number of daily steps measured with a StepWatch Activity Monitor. Secondary outcomes include sleep, behaviour and quality of life. Caregiver experiences will be assessed immediately after the intervention using a satisfaction questionnaire. Group differences for each outcome will be evaluated with analysis of covariance, adjusting for baseline values on an intention-to-treat basis.

Ethics and dissemination Ethics approval has been obtained in Western Australia from the Child and Adolescent Health Services (RGS3371), in Denmark from the Capital Region Ethics Committee $(\mathrm{H}-19040514)$ and in Israel from the Ariel University Institutional Review Board (AU-HEA-ML-20190331). Manuscripts on the development of the intervention from pilot work and the results of the intervention will be submitted to peer-reviewed journals. Results will be presented at conferences and consumer forums. We will develop an online resource documenting the physical activity programme and available supporting evidence.

Trial registration number NCT04167059; Pre-results.
Strengths and limitations of this study

- This study will use clinical trial methods to evaluate an intervention delivered via telehealth to increase objectively measured physical activity in individuals with Rett syndrome (RTT).

- Activities will be determined by goals that are set, modified and developed in collaboration with caregivers, ${ }^{42}$ and based on contemporary models for participation $^{37}$ and quality of life..$^{20} 38$

- Recruitment will be non-probabilistic, and the sample may not be representative of the population of RTT with some walking skills.

- We acknowledge that our findings will not inform how to increase activity in individuals who are unable to walk.

- The waitlist control condition may bias the study in favour of the intervention group because the control group could be less likely to initiate changes in lifestyle.

\section{INTRODUCTION}

Rett syndrome (RTT, MIM:312750) is a rare neurodevelopmental disorder affecting approximately 1 in 9000 live female births. ${ }^{1}$ It is characterised by largely normal early development followed by loss of acquired hand and communication skills. There are four main criteria: loss of hand skills, loss of communication skills, hand stereotypies and gait abnormalities. ${ }^{2}$ RTT is caused by a mutation in the X-linked MECP2 gene, ${ }^{3}$ it is a severe neurodevelopmental disorder ${ }^{4}$ impacting multiple body systems $\mathrm{s}^{5-7}$ and associated with severe functional impairments. The clinical spectrum is broad and varied and closely linked to the type of genetic mutation. ${ }^{8-10}$ The clinical presentation is severe for some children who are unable to walk or go on to require enteral feeding, whereas it is 
milder for others who can walk, negotiate stairs and selffeed. Communication is most often non-verbal, although a range of abilities is seen. Across the literature, findings are consistent that much of the clinical spectrum relates to the type of genetic mutation. For example, the Arg270*, Arg255* and Arg168* mutations are associated with more severe symptoms, whereas the Arg133Cys, Arg294* and C-terminal deletions are associated with less severe symptoms. ${ }^{11-14}$

Specifically, early gross motor impairments may include delay in the acquisition of gross motor skills prior to regression, ${ }^{15}$ loss of balance during the regression period ${ }^{16}$ and difficulty in walking. ${ }^{2}$ Gross motor skills are variable ${ }^{12}$; the majority of individuals are able to sit, fewer than half learn to walk independently and less than one quarter can transfer from sitting to standing independently. ${ }^{17}$ Recent systematic reviews of physical therapy interventions for individuals with RTT have reported mostly positive effects on gross motor skills, motor endurance and fitness within therapy settings. ${ }^{18-20}$ Opportunities for training have been observed as enjoyable for the child when accompanied with a microswitch that delivered a light and sound sensory response to walking. ${ }^{21}$ We note that the majority of studies were case studies, case series and single-case design studies, and only one study used randomisation in a stepped wedge design. ${ }^{22}$

Use of gross motor skills as physical activity during everyday living is also an important component of health ${ }^{23}$ and quality of life. ${ }^{24}$ We have previously investigated sedentary time and physical activity in RTT. ${ }^{25-28}$ Girls and women recruited from the Australian Rett Syndrome Database (ARSD, $n=64)^{25} 26$ wore a StepWatch Activity Monitor (SAM) during waking hours. An average of $62 \%$ (SD 19\%) of time was sedentary and approximately $20 \%$ (SD $8 \%$ ) of time was spent in light-intensity walking, with the volume of activity influenced by whether walking was independent or required assistance or even close supervision, older age and the presence of comorbidities such as epilepsy and scoliosis. ${ }^{27}$ We found similar results in Denmark, again using the activPAL to measure sedentary time and the SAM to count steps ${ }^{28}$ On average, $83 \%$ of waking hours were sedentary $(n=48)$, the median daily step count was $5127(\mathrm{n}=28)$ and older age and lower levels of gross motor skill were strong predictors of reduced activity levels. ${ }^{28}$

Individuals with RTT are mostly dependent on others for support in daily activities. ${ }^{29}{ }^{30}$ Providing support for the individual to engage in additional activities could reduce sedentary time and increase physical activity. Reducing sedentary time by increasing the number and duration of episodes of standing and walking, especially in individuals with more severe mobility limitations, has been suggested. ${ }^{31}$ Providing support for more light activities in RTT could be an important focus for intervention. More recently, we evaluated the feasibility and effectiveness of an individualised 12-week intervention using a pretest-posttest design that aimed to increase standing and walking activity in 14 girls and women with RTT. ${ }^{32}$ The intervention involved working with caregivers and service providers to identify standing and walking activities using strategies developed to meet activity goals $^{33}$ and that were consistent with the concept of participation in home, school and community settings. ${ }^{34}$ An important focus was the collaborative efforts with families in selecting meaningful and engaging goals and in implementing and evaluating the individual intervention plan. It was well received and considered feasible by caregivers and therapists. Some positive effects were found including reduced sedentary time, increased daily step count and a small but significant increase in quality of life. ${ }^{32}$

In sum, individuals with RTT live sedentary lives and engage in low levels of physical activity relative to the general population, which has shown to reduce functional abilities throughout adulthood in people with disabilities. ${ }^{35}$ Programmes of support for increasing participation in meaningful activities are feasible and have been proven to increase function, ${ }^{36}$ but the level of evidence is low. As RTT is a rare condition, individuals are geographically dispersed but telehealth methods are being used increasingly across distances for the delivery of rehabilitation services such as for individuals diagnosed with cerebral palsy and traumatic brain injury ${ }^{37}$ and across a range of domains to improve adaptive skills in those with multiple disabilities. ${ }^{38}$ To build on previous research and inform practice, we designed this ActivRett clinical trial protocol.

The primary aim is to evaluate the effects of a telehealthdelivered physical activity programme on sedentary behaviour and physical activity in RTT. Our secondary aims are to evaluate the effects of the programme on quality of life, sleep and behaviour. We will also seek to evaluate the acceptability of the physical activity programme for caregivers and to explore the characteristics of individuals who are more responsive to the programme and the adaptability of activities in accordance with family resources. Following trial completion, we aim to provide the study manual as an attractive and freely available online resource for physical activity support in RTT, for use by families, caregivers and therapists wherever they live.

\section{METHODS AND ANALYSIS}

\section{Study design}

A randomised waitlist-controlled trial will be implemented. Participants who are randomised to control conditions during the first time period will receive the intervention during the second time period. Outcomes will be assessed at baseline, immediately following a 12-week intervention period and then 12 weeks after the second intervention period. An overview of the study design is shown in figure 1 . The trial is registered with ClinicalTrials.gov and summary data are presented in table 1 .

\section{Participant and public involvement}

This study is funded by the peak international patient advocacy organisation for RTT (Rettsyndrome.org) and 


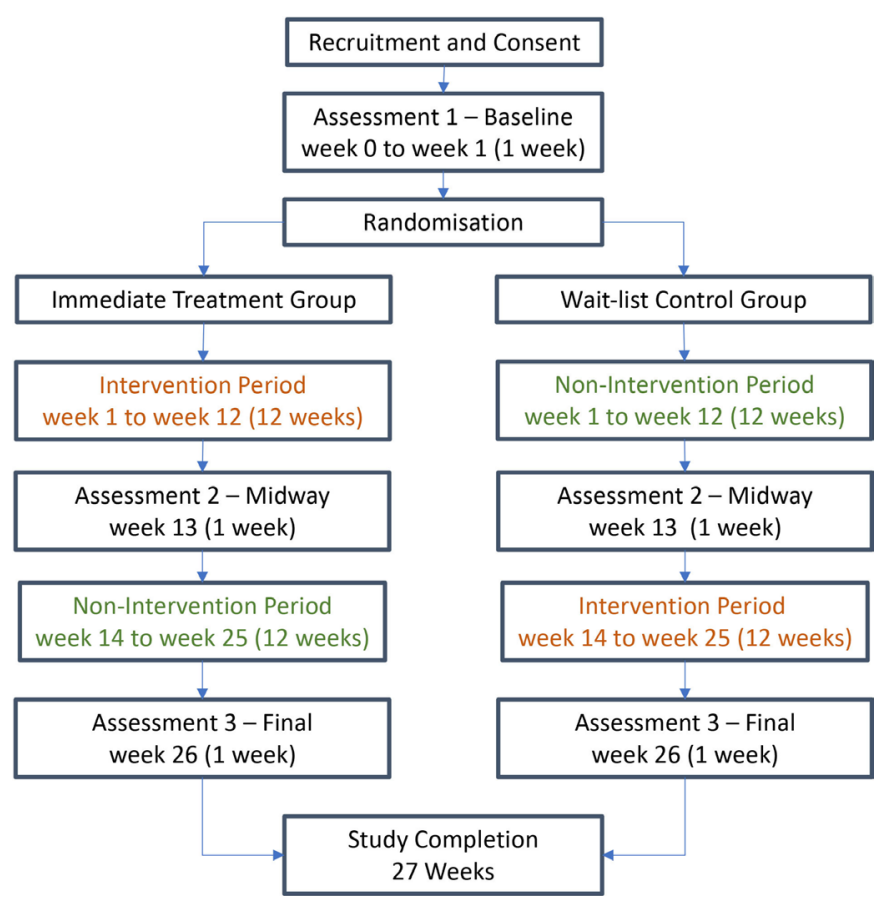

Figure 1 Participant flow through the study.

our proposal has been subject to scientific and consumer review. Prior to our previous feasibility study, ${ }^{32}$ we held a teleconference with Australian parents who shared their experiences of enjoyable physical activities for their daughters. When developing our manual for the current study, we conducted an international survey of caregivers and professionals who support individuals with RTT about how they supported their daughter to be physically active and what they would want in a physical activity programme. These ideas and perspectives informed the study intervention manual. Insights from the survey will be published to share with parents and therapists key insights on facilitators and barriers to individuals with RTT being physically active. The dissemination plan will include presentations to families at consumer and community forums for their feedback.

\section{Participants and settings}

Individuals aged 5years and older with genetically confirmed RTT and who live in either Australia, Denmark or Israel will be recruited to the study, commencing the beginning of 2020. Some will be able to walk independently, and others will need assistance. We will exclude individuals who are unable to walk because our intervention will be provided remotely, and we are unable to organise or provide participants with the necessary equipment such as a standing frame within the time frame of this study. We will also exclude participants who have had a spinal fusion over the previous 12 months or lower extremity orthopaedic surgery over the previous 6 months.

For this study, we will recruit from three sources:

1. The ARSD is an ongoing population-based longitudinal study of RTT which was established in 1993 and
Table 1 Summary of the WHO Trial Registration Data Set for the ActivRett trial

\section{Data category}

\begin{tabular}{ll}
$\begin{array}{l}\text { Primary registry and trial } \\
\text { identifying number }\end{array}$ & $\begin{array}{l}\text { ClinicalTrials.gov } \\
\text { (NCT04167059) }\end{array}$ \\
\hline Protocol version & V.4, 3 October 2019 \\
$\begin{array}{l}\text { Date of registration in primary } \\
\text { registry }\end{array}$ & 18 November 2019 \\
\hline
\end{tabular}

Source of monetary or material Rettsyndrome.org support

\begin{tabular}{|c|c|}
\hline Primary sponsor & Rettsyndrome.org \\
\hline Contact for public queries & JD, email address \\
\hline Contact for scientific queries & JD, email address \\
\hline Public title & ActivRett \\
\hline Scientific title & $\begin{array}{l}\text { Implementing telehealth } \\
\text { support to increase physical } \\
\text { activity in girls and women with } \\
\text { Rett syndrome }\end{array}$ \\
\hline Countries of recruitment & Australia, Denmark, Israel \\
\hline Problem being studied & $\begin{array}{l}\text { Low levels of physical activity } \\
\text { in individuals with Rett } \\
\text { syndrome }\end{array}$ \\
\hline \multirow[t]{2}{*}{ Intervention } & $\begin{array}{l}\text { Active comparator: } \\
\text { participation-based activity } \\
\text { programme aiming to increase } \\
\text { standing and walking activities }\end{array}$ \\
\hline & $\begin{array}{l}\text { Control comparator: usual } \\
\text { activity }\end{array}$ \\
\hline \multirow[t]{2}{*}{$\begin{array}{l}\text { Key inclusion and exclusion } \\
\text { criteria }\end{array}$} & $\begin{array}{l}\text { Ages eligible for study: } 5 \\
\text { years and older; genetically } \\
\text { confirmed Rett syndrome; } \\
\text { female because Rett syndrome } \\
\text { mostly effects females }\end{array}$ \\
\hline & $\begin{array}{l}\text { Inclusion criteria: able to } \\
\text { walk independently or with } \\
\text { assistance }\end{array}$ \\
\hline
\end{tabular}

Exclusion criteria: spinal fusion over the previous 12 months; lower extremity orthopaedic surgery over the previous 6 months

\begin{tabular}{ll|}
\hline Study type & $\begin{array}{l}\text { Interventional } \\
\text { Randomised waitlist-controlled } \\
\text { trial }\end{array}$ \\
& $\begin{array}{l}\text { Primary purpose: prevention } \\
\text { Phase III }\end{array}$ \\
\hline Date of first enrolment & 7 January 2020 \\
\hline Target sample size & 60 \\
\hline Recruitment status & Recruiting \\
\hline Primary outcomes & Number of steps per day; per \\
& cent of sedentary time \\
\hline Key secondary outcomes & Quality of life; sleep; behaviour \\
\hline
\end{tabular}

Key secondary outcomes Quality of life; sleep; behaviour

includes individuals with genetically confirmed and clinical RTT born since $1976 .{ }^{26}{ }^{39}$ Clinical and multiple waves of family-reported data are stored in the database. 
2. The Danish Center for Rett syndrome was established in 2007 and offers counselling and annual follow-up across the lifespan of all individuals diagnosed with RTT. ${ }^{30}$ Clinical and medical data are stored in the Danish Rett syndrome database.

3. The Rett Syndrome Association of Israel was established in 2001 and is in contact with approximately 200 individuals diagnosed with RTT.

Families will be contacted by telephone by the site investigator for initial discussion of the study and invited to participate if it is established that they are eligible. Individuals with RTT are presumed to have cognitive impairment and are unable to provide informed consent to participate in the study. As is usual in the population, informed consent will be collected from the parents/legal guardians of the individual (see online supplemental file $1)$.

\section{Randomisation}

Allocation concealment will be ensured by performing allocation after completing baseline assessments (figure 1). At each site, individuals will be stratified by walking ability (independent or assisted) and then randomised using computer-generated randomisation (V.14.1, StataCorp, College Station, Texas, USA) to the immediate treatment group or waitlist control group. A statistician independent to other study processes is responsible for group allocation and communicating this to the site investigators.

\section{Intervention procedures}

Contact for the intervention sessions will be made using the telephone and Skype or Zoom platforms (figure 2). The initial intervention session will involve review of the gross motor skills, care routines, participation activities as well as physical surrounding opportunities of the individual with RTT and discussion of the activity patterns with the caregiver and other service providers as appropriate. If several caregivers are involved, we will aim for a joint meeting at the beginning of the intervention with subsequent contacts separately or together, as is feasible.

The intervention aims to increase the quantity of standing and walking activities, collectively termed 'uptime', ${ }^{40}$ for girls and women with RTT. The increased 'uptime' activities will be codesigned with the caregivers and service providers for use in their own environments (home, school, community) and supported by the usual caregivers and service providers in those environments. These will primarily be parents, but may also include others such as siblings, carers and service providers. The intervention will be delivered over a 12-week period, supported by fortnightly telehealth video/telephone calls with a physiotherapist (the site investigators) who will consult and provide recommendations for strategies to increase the quantity and frequency of 'uptime' activities. The activity recommendations will be formed into goals and evaluated at the next session using Goal Attainment Scaling. ${ }^{41}$ The research therapists will also be available between scheduled fortnightly video calls for

\section{Community input into ActivRett program design}

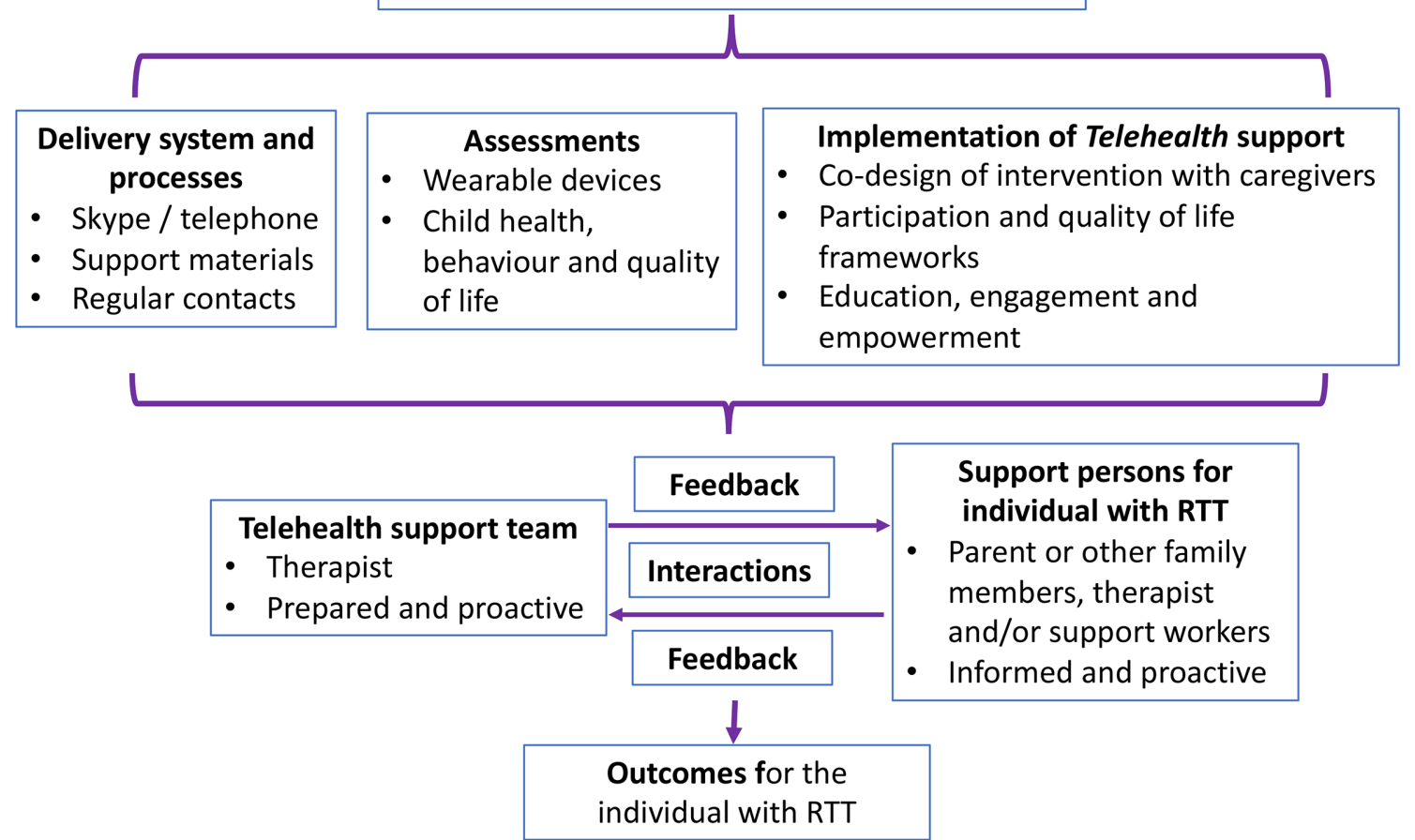

Figure 2 Summary of the components of the assessment and telehealth delivered intervention, and iterative feedback processes over the intervention period. 
support via email, phone call or video call. Throughout, the investigators and carers will discuss physical activity needs, determine and adapt relevant activities, modify the programme as necessary and plan additional goals. For example, goals will articulate what the activity is (eg, increased opportunities for standing or walking as suitable for the individual's ability), where (eg, in the home, garden, at school or in the community) and when the activity will happen (eg, the new activity could be scheduled at particular times in the day to suit routines) and with whom (eg, parent, sibling, carer) and why the goal is meaningful and of value. At any time during the intervention period, an individual will have two to four goals which could change, as more suitable and challenging goals are identified. The interactive nature of the delivery of the programme is illustrated in figure 2.

The intervention strategies will be consistent with contemporary participation, ${ }^{42}$ quality of life ${ }^{24} 43$ and goal setting frameworks for disability.

1. Optimal participation in recreational activities involves an interaction and balance between the dimensions and determinants of participation. ${ }^{42}$ Dimensions of participation include: (1) physical engagementdoing the activity. Being actively involved underlies participation and has positive impacts on fitness and physical health; (2) social engagement-interactions and feeling included. A sense of belonging defines successful and meaningful participation; and (3) self-engagement-enjoyment and choice-making. Enjoyment is paramount to engagement in activities and through choice people can identify preferred activities. ${ }^{42}$ Determinants of participation include: (1) child-related attributes-combination of body function and personal factors, (2) family-related and caregiver-related attributes-socioeconomic status and family functioning, and (3) environmental attributes-supportive of the physical, social, attitudinal and service environments. ${ }^{42}$

2. Quality of life domains identified as important for individuals with RTT include aspects of physical and mental well-being and social and recreational functioning. More specifically, the quality of life factors include 'physical health', 'positive emotions', 'negative emotions', 'social interaction', 'leisure and the outdoors' and 'independence ${ }^{43}$ and are consistent with the International Classification of Functioning, Disability and Health (ICF) structure. ${ }^{44}$

3. Achievement of goals will guide the development of ongoing programme activities. Meaningful activity goals will be identified with carers at the baseline assessment, informed by participation ${ }^{45}$ and quality of life ${ }^{43}$ models and measured using Goal Attainment Scaling. ${ }^{46}$ The setting and review of goals is recognised as supporting the learning of new routines and health behaviours. ${ }^{47}$

Usual care comprising medical care and therapy activities will continue during the intervention and control periods.

\section{Intervention consistency}

Each site investigator participated in the development of the study manual and was familiar with the delivery of the assessment and intervention protocols. The site investigators meet regularly to discuss implementation of the protocol and any problems that arise. This is to ensure intervention consistency within and across sites.

\section{Adverse event reporting}

At each contact, investigators delivering the intervention will check with the caregiver as to the well-being of the individual with RTT and how the intervention activities have progressed. The intervention aims to increase the volume of everyday activities under the supervision of primary caregivers and there is the risk of a fall. Any adverse events will be reported to the study data safety monitor, a paediatric orthopaedic surgeon and the ethics committee. The data safety monitor will use clinical judgement as to the circumstances around any adverse events and will advise whether the individual can continue in the trial or not. Participants who have an adverse event will be followed up by the site investigator as required to ensure they are receiving appropriate medical care.

RTT is associated with increased risk of fracture and we have previously determined that the incidence of any fracture episode was 43.3 episodes per 1000 years. ${ }^{48}$ We will be observing 60 individuals with RTT over a period of 6 months per individual, and this would equate to 30 person years. We would expect that up to two episodes of fracture could occur during the study period, associated with either activity or a pathological fracture. The stopping rule for this study will be the occurrence of two episodes of fracture that are associated with a study activity-related fall. The risk of adverse events is low and a data monitoring committee is not needed, additional to the data safety monitor, for this study.

\section{Data collection}

Data will be provided to Telethon Kids Institute using REDCap which is maintained and administered by the biostatistics team at Telethon Kids Institute, who are Good Clinical Practice compliant. The system runs on secure (password-protected), encrypted servers located at Telethon Kids with offsite backup. For analysis, data will be downloaded from REDCap to Excel and thereafter to STATA statistical analysis software. Participant's information and outcome measures are summarised in the schedule of assessments (table 2). Data will be checked regularly for outliers and missing data. If an outlier is found, the site investigator will attempt to check whether the value is likely or not. It will be included unless it is identified as very unlikely. If missing data are found, the Investigator will attempt to collect the missing value.

\section{Participant characteristics}

At the beginning of the study, parents will be asked about their child's gross motor abilities, which will inform the completion of the Rett Syndrome Gross Motor Scale ${ }^{12}$ by 
Table 2 SPIRIT schedule of enrolment, interventions and assessment

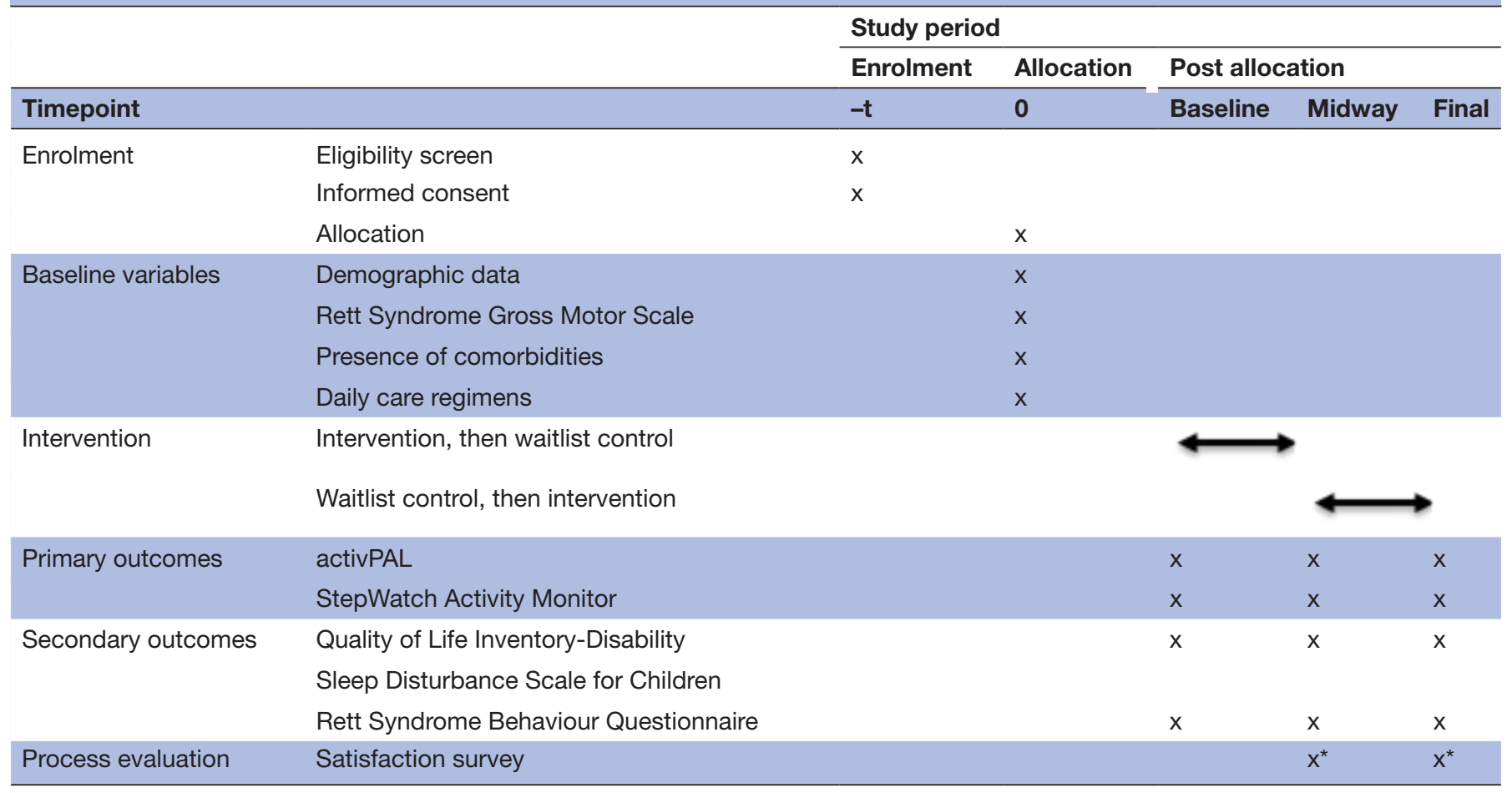

*Satisfaction survey is administered post intervention, which will be either at the midway or final assessment, depending on randomisation to the intervention or non-intervention group for the first 12-week block.

the investigators. Daily care regimens and activities will be documented and participation will be measured using the Participation and Environment Measure for Children and Youth, ${ }^{49}$ to guide the design of the intervention. To describe other aspects of health and well-being, diagnosed comorbidities will be described.

\section{Primary outcome measures}

Suitable outcome measures with validation data for RTT were selected. ${ }^{50}$

1. The primary outcome of sedentary time will be measured using the activPAL. The activPAL (PAL Technologies, Glasgow, UK) is a small $(53 \times 35 \times 7 \mathrm{~mm}$, $20 \mathrm{~g}$ ) uniaxial accelerometer which is attached to the skin on the anterior thigh with an adhesive pad. It contains an inclinometer which determines the position of the thigh and estimates the time spent in a sitting/ lying or standing position in $15 \mathrm{~s}$ epochs. We have validated its use as a measure of sedentary time in RTT. ${ }^{51}$ Four valid days of data including 1 weekend day will be required for analysis. For each day and guided by diary data indicating awake hours out of bed, we will calculate the per cent of time in uptime activities, that is, upright activities of standing and/or walking, of the total awake hours out of bed. Mean values across each of the valid days will be calculated. ${ }^{51}$ This will be calculated using data collected for a 1-week period at the baseline, mid and final assessment.
2. Daily steps will be measured using the SAM. The SAM (Modus Health LLC, Washington, DC) is a small $(70 \times 50 \times 20 \mathrm{~mm}, 38 \mathrm{~g})$ accelerometer-type device that records the number of steps taken every minute. It is attached to the ankle using an elastic Velcro strap. We have validated the SAM as a measure of daily steps in individuals with RTT. ${ }^{52}$ Four valid days of data including 1 weekend day will be required for analysis. These assessments have also been validated against a proxyreport measure of 'uptime' (total time standing and walking) and 'downtime' (lying and sleeping) using the modified Bouchard activity record. ${ }^{40} 51$ For each day and guided by diary data indicating hours out of bed, we will calculate the number of steps for total awake time. Mean values across each of the valid days will be calculated. This will be calculated using data collected for a 1-week period at the baseline, mid and final assessment.

\section{Secondary outcome measures}

The secondary outcome of quality of life will be measured using the Quality of Life Inventory for Disability, ${ }^{43}$ sleep will be measured with the Disorders of Initiating and Maintaining Sleep and the Disorders of Excessive Somnolence subscales of the Sleep Disturbance Scale for Children $^{53}$ and behaviour will be measured with the Mood and the Fear and Anxiety subscales of the Rett Syndrome Behavior Questionnaire. ${ }^{54}$ 


\section{Caregiver satisfaction}

Carers and parents will complete a study-specific satisfaction questionnaire immediately following the intervention to document how the programme was received, enablers and barriers when implementing the programme and other impacts on daily life.

\section{Data analysis}

\section{Hypothesis testing}

Data related to sedentary behaviour, step counts, sleep, behaviour and quality of life at each assessment will be described and visualised to illustrate levels and trends. $\chi^{2}$ test and Student's t-test will be used for categorical and continuous variables, respectively, to detect differences in baseline characteristics between the immediate treatment and waitlist control groups. Difference in values of each outcome variable by intervention status at assessment 2 will be analysed using analysis of covariance, adjusted for baseline values, age, walking status and country. Analyses will be on an intention-to-treat basis with the last known value carried forward in the event of missing data. Further within-group analyses on treatment effect will be conducted by combining data collected immediately pre intervention and immediately post intervention from both groups. In this analysis, the independent variables will be factors that could influence the magnitude of change, such as age and walking ability, and the dependent variables will be the change scores of the primary outcomes (ie, sedentary time and step counts). Univariate and multivariate linear regression models will be conducted. For the group which receives the intervention in the first time period, we will evaluate change in outcomes between assessment 2 and assessment 3 using a paired t-test. The analyst will be blind to treatment allocation.

\section{Sample size}

In preliminary Danish data, ${ }^{32}$ the response of individuals to the physical activity programme (per cent of sedentary behaviour) in a repeated measures design was normally distributed with an SD of $11 \%$. If the true reduction, following the intervention, in the mean per cent of sedentary time is $5 \%$ and assuming a pairwise correlation of 0.5 , we will need to study 50 individuals in a twosample trial to be able to reject the null hypothesis that this response difference is 0 , with probability (power) 0.8 . The type I error probability associated with this test of the null hypothesis is 0.05 . We will recruit 60 families, allowing for some dropout. A total of 26 individuals will be recruited from Australia, 14 from Denmark and 20 from Israel.

\section{Caregiver satisfaction}

Satisfaction survey questions are rated on a 1-10 Visual Analogue Scale and will be described and graphed to illustrate frequency distributions to identify positive and negative aspects of the study processes.

\section{ETHICS AND DISSEMINATION}

\section{Ethics approval}

Ethics approval has been obtained from the Child and Adolescent Health Services (RGS3371) in Australia, the Capital Region Ethics Committee in Denmark (H-19040514) and the Ariel University Institutional Review Board (AU-HEA-ML-20190331) in Israel. Protocol amendments will be submitted to each ethics committee.

\section{Dissemination}

We plan to submit a manuscript on the development of the intervention and a manuscript of the trial results to a peer-reviewed journal. Results will be presented at conferences and consumer forums. Following the conclusion of the study, a website will be developed that presents strategies of how to evaluate and support individuals with RTT to live physically active lives. It will contain introductory information about how the current project was conducted, the participation and quality of life frameworks that inform how to address physical activity needs and the findings from the current proposal. Information will be suitable for families, caregivers and therapists, and will also be suitable for a wide range of disabilities in addition to RTT. Strategies will be illustrated with line drawings. Draft content will be presented to the Consumer Reference Groups of the Australian Rett Syndrome Study and InterRett, open also to Danish and Israeli families, for review prior to being finalised and translated. In the first instance, we will produce the website in English, Danish and Hebrew. The database infrastructures, family advocacy associations and the institutions and networks of the investigators will support us with dissemination strategies.

\section{DISCUSSION}

Many individuals with a developmental disability including those with RTT experience low levels of physical activity in their daily lives, threatening both their health and quality of life. There is a need for scalable, flexible and accessible interventions to promote activity programmes in RTT. Yet, the current level of evidence for interventions that guide how to achieve this is low. This study will be the first clinical trial investigating strategies to increase physical activity in RTT. Participants will be children and adults. The literature over the years has suggested the need to develop management plans that can translate from research designs to the daily life of the participants and across their life span. ${ }^{55-57}$ Our proposal represents affirmative action in this regard.

Telehealth is being used in many fields of medicine and rehabilitation and our proposal will be one of the first to apply this method in RTT. This is a particularly attractive modality because RTT is rare, families live in widespread geographic locations and telehealth can bring specialist skills to the affected individual in her own environment. Particularly, in light of the social isolation and disruption to traditional therapy delivery caused by the ongoing COVID-19 pandemic, telehealth represents an attractive 
method to provide therapy to populations that are likely to be disproportionately negatively affected, such as individuals with RTT and their caregivers. Families, carers and therapists will guide the setting of goals and be responsible for implementing activities that they have had a role in determining. Their grass roots involvement will facilitate the continuation of programmes beyond the immediate study period. Our proposal will be build on available resources and strengths, respond to locally identified needs and empower those working in local communities to improve physical activities and participation of the individuals with RTT under their care.

The intervention is in alignment with participation ${ }^{58}$ and quality of life ${ }^{24}$ frameworks, developed by our team of international investigators, to enable the intervention to be applicable across diverse settings. As follow-up to the proposed evaluation, there is also a critical need to develop mechanisms by which evidence-based practice can be readily available to the community. We also propose the translation of available strategies including those tested in the current study and their level of evidence into an online resource, in order that physical activity strategies are available to those in need wherever they live. There is potential for the findings of our study to provide evidence of how individuals with RTT can live more active lives, in order that they may gain health and well-being benefits from greater levels of meaningful activities, within their home, school and community environments.

\section{Author affiliations \\ ${ }^{1}$ Telethon Kids Institute, The University of Western Australia, Nedlands, Western Australia, Australia \\ ${ }^{2}$ School of Physiotherapy and Exercise Science, Curtin University, Perth, Western Australia, Australia \\ ${ }^{3}$ Department of Physiotherapy, Ariel University, Ariel, Israel \\ ${ }^{4}$ School of Creative Arts Therapies, University of Haifa, Haifa, Israel \\ ${ }^{5}$ Department of Paediatrics and Adolescent Medicine, Center for Rett Syndrome, Copenhagen, Denmark}

\section{Twitter Jenny Downs @drjennydowns}

Acknowledgements The authors would like to thank all families, group home caregivers and professionals who participated in our ongoing research programs on Rett syndrome. We also acknowledge and thank the Australian Paediatric Surveillance Unit and the Rett Syndrome Association of Australia for their ongoing support in case ascertainment. The authors would also like to thank the Rett Syndrome Association of Israel and the Danish Center for Rett Syndrome for supporting the implementation of the current research project. The Australian Rett Syndrome Study was previously supported by the National Institute of Health Grant 5R01HD043100-05 and National Health and Medical Research Council (NHMRC) project grants \#303189, \#1004384 and \#1103745. HL is funded by an NHMRC Senior Research Fellowship (\#1117105).

Contributors JD led the study conceptualisation and the first draft of the proposal and development of intervention, and is leading the study. ML, CE, $\mathrm{HL}$ and MS contributed to study conceptualisation, development of intervention content and edited the protocol. KW designed the analysis plan and edited the protocol. NB contributed to the development of intervention content and edited the protocol. All authors edited and approved the final manuscript.

Funding This work is supported by funding from Rettsyndrome.org, grant number \#3607 (https://www.rettsyndrome.org). The funder will not influence the decisionmaking for the study design; collection, management, analysis and interpretation of data; the writing of the report and the decision to submit the report for publication.

Competing interests JD is employed at Telethon Kids Institute where she is program head of Child Disability. The protocol for this study was developed for this grant received from Rettsyndrome.org. The authors do not have competing interests to declare in relation to this study.

Patient consent for publication Not required.

Provenance and peer review Not commissioned; externally peer reviewed.

Supplemental material This content has been supplied by the author(s). It has not been vetted by BMJ Publishing Group Limited (BMJ) and may not have been peer-reviewed. Any opinions or recommendations discussed are solely those of the author(s) and are not endorsed by BMJ. BMJ disclaims all liability and responsibility arising from any reliance placed on the content. Where the content includes any translated material, BMJ does not warrant the accuracy and reliability of the translations (including but not limited to local regulations, clinical guidelines, terminology, drug names and drug dosages), and is not responsible for any error and/or omissions arising from translation and adaptation or otherwise.

Open access This is an open access article distributed in accordance with the Creative Commons Attribution Non Commercial (CC BY-NC 4.0) license, which permits others to distribute, remix, adapt, build upon this work non-commercially, and license their derivative works on different terms, provided the original work is properly cited, appropriate credit is given, any changes made indicated, and the use is non-commercial. See: http://creativecommons.org/licenses/by-nc/4.0/.

\section{ORCID iDs}

Jenny Downs http://orcid.org/0000-0001-7358-9037

Helen Leonard http://orcid.org/0000-0001-6405-5834

\section{REFERENCES}

1 Fehr S, Bebbington A, Nassar N, et al. Trends in the diagnosis of Rett syndrome in Australia. Pediatr Res 2011;70:313-9.

2 Neul JL, Kaufmann WE, Glaze DG, et al. Rett syndrome: revised diagnostic criteria and nomenclature. Ann Neurol 2010;68:944-50.

3 Amir RE, Van den Veyver IB, Wan M, et al. Rett syndrome is caused by mutations in X-linked MeCP2, encoding methyl-CpG-binding protein 2. Nat Genet 1999;23:185-8.

4 Anderson A, Wong K, Jacoby P, et al. Twenty years of surveillance in Rett syndrome: what does this tell us? Orphanet $J$ Rare Dis 2014;9:87.

5 Bao X, Downs J, Wong K, et al. Using a large international sample to investigate epilepsy in Rett syndrome. Dev Med Child Neurol 2013:55:553-8.

6 Jian L, Nagarajan L, de Klerk N, et al. Seizures in Rett syndrome: an overview from a one-year calendar study. Eur J Paediatr Neurol 2007;11:310-7.

7 Mackay J, Downs J, Wong K, et al. Autonomic breathing abnormalities in Rett syndrome: caregiver perspectives in an international database study. J Neurodev Disord 2017;9:15.

8 Bebbington A, Anderson A, Ravine D, et al. Investigating genotypephenotype relationships in Rett syndrome using an international data set. Neurology 2008;70:868-75.

9 Cuddapah VA, Pillai RB, Shekar KV, et al. Methyl-CpG-binding protein 2 (MECP2) mutation type is associated with disease severity in Rett syndrome. J Med Genet 2014;51:152-8.

10 Leonard H, Cobb S, Downs J. Clinical and biological progress over 50 years in Rett syndrome. Nat Rev Neurol 2017;13:37-51.

11 Bebbington A, Anderson A, Ravine D, et al. Investigating genotypephenotype relationships in Rett syndrome using an international data set. Neurology 2008;70:868-75.

12 Downs J, Stahlhut M, Wong K, et al. Validating the Rett syndrome gross motor scale. PLoS One 2016;11:e0147555.

13 Fabio RA, Caprì T, Lotan M. Motor abilities are related to the specific genotype in Rett syndrome. In: Advances in genetics research. New York: Nova Science Publisher, 2018.

14 Neul JL, Fang P, Barrish J, et al. Specific mutations in methylCpG-binding protein 2 confer different severity in Rett syndrome. Neurology 2008;70:1313-21.

15 Fehr S, Bebbington A, Ellaway C, et al. Altered attainment of developmental milestones influences the age of diagnosis of Rett syndrome. J Child Neurol 2011;26:980-7.

16 Lee JYL, Leonard H, Piek JP, et al. Early development and regression in Rett syndrome. Clin Genet 2013;84:572-6.

17 Downs JA, Bebbington A, Jacoby P, et al. Gross motor profile in Rett syndrome as determined by video analysis. Neuropediatrics 2008;39:205-10.

18 Amoako AN, Hare DJ. Non-Medical interventions for individuals with Rett syndrome: a systematic review. J Appl Res Intellect Disabil 2020;33:808-27. 
19 Fonzo M, Sirico F, Corrado B. Evidence-Based physical therapy for individuals with Rett syndrome: a systematic review. Brain $\mathrm{Scl}$ 2020;10 doi:10.3390/brainsci10070410

$20 \mathrm{Lim}$ J, Greenspoon D, Hunt A, et al. Rehabilitation interventions in Rett syndrome: a scoping review. Dev Med Child Neurol 2020;62:906-16.

21 Stasolla F, Caffò AO, Perilli V, et al. Fostering locomotion fluency of five adolescents with Rett syndrome through a Microswitch-Based program: contingency awareness and social rating. J Dev Phys Disabil 2018;30:239-58.

22 Downs J, Rodger J, Li C, Chen L, et al. Environmental enrichment intervention for Rett syndrome: an individually randomised stepped wedge trial. Orphanet J Rare Dis 2018;13:3.

23 MacKay J, Leonard $\mathrm{H}$, Wong $\mathrm{K}$, et al. Respiratory morbidity in Rett syndrome: an observational study. Dev Med Child Neurol 2018;60:951-7.

24 Epstein A, Leonard H, Davis E, et al. Conceptualizing a quality of life framework for girls with Rett syndrome using qualitative methods. Am J Med Genet A 2016;170:645-53.

25 Laurvick CL, de Klerk N, Bower C, et al. Rett syndrome in Australia: a review of the epidemiology. J Pediatr 2006;148:347-52.

26 Leonard H, Bower C, English D. The prevalence and incidence of Rett syndrome in Australia. Eur Child Adolesc Psychiatry 1997;6:8-10.

27 Downs J, Leonard H, Wong K, et al. Quantification of walkingbased physical activity and sedentary time in individuals with Rett syndrome. Dev Med Child Neurol 2017;59:605-11.

28 Stahlhut M, Downs J, Aadahl M. Patterns of sedentary time and ambulatory physical activity in a Danish population of girls and women with Rett syndrome. Disabil Rehabil 2017:1-9.

29 Leonard H, Fyfe S, Leonard S, et al. Functional status, medical impairments, and rehabilitation resources in 84 females with Rett syndrome: a snapshot across the world from the parental perspective. Disabil Rehabil 2001;23:107-17.

30 Schönewolf-Greulich B, Stahlhut M, Larsen JL, et al. Functional abilities in aging women with Rett syndrome - the Danish cohort. Disabil Rehabil 2017;39:911-8.

31 Verschuren O, Darrah J, Novak I, et al. Health-Enhancing physical activity in children with cerebral palsy: more of the same is not enough. Phys Ther 2014;94:297-305.

32 Stahlhut M, Downs J, Wong K, et al. Feasibility and Effectiveness of an Individualized 12-Week 'Uptime' Participation (U-PART) Intervention in Girls and Women With Rett Syndrome. Phys Ther 2020;100:168-79.

33 Palisano RJ, Chiarello LA, King GA, et al. Participation-based therapy for children with physical disabilities. Disabil Rehabil 2012;34:1041-52.

34 Imms C, Adair B, Keen D, et al. 'Participation': a systematic review of language, definitions, and constructs used in intervention research with children with disabilities. Dev Med Child Neurol 2016;58:29-38.

35 Peterson MD, Gordon PM, Hurvitz EA. Chronic disease risk among adults with cerebral palsy: the role of premature sarcopoenia, obesity and sedentary behaviour. Obes Rev 2013;14:171-82.

36 Lotan M, Isakov E, Merrick J. Improving functional skills and physical fitness in children with Rett syndrome. J Intellect Disabil Res 2004;48:730-5.

37 Hailey D, Roine R, Ohinmaa A, et al. The status of telerehabilitation in neurological applications. J Telemed Telecare 2013;19:307-10.

38 Caprì T, Nucita A, lannizzotto G, et al. Telerehabilitation for improving adaptive skills of children and young adults with multiple disabilities: a systematic review. Review Journal of Autism and Developmental Disorders 2020;9.

39 Downs J, Torode I, Wong K, et al. The natural history of scoliosis in females with Rett syndrome. Spine 2016;41:856-63.

40 Lor L, Hill K, Jacoby P, et al. A validation study of a modified Bouchard activity record that extends the concept of 'uptime' to Rett syndrome. Dev Med Child Neurol 2015;57:1137-42.

41 Kiresuk TJ, Sherman RE. Goal attainment scaling: a general method for evaluating comprehensive community mental health programs. Community Ment Health J 1968;4:443-53.

42 Kang L-J, Palisano RJ, King GA, et al. A multidimensional model of optimal participation of children with physical disabilities. Disabil Rehabil 2014;36:1735-41.

43 Downs J, Jacoby P, Leonard H, et al. Psychometric properties of the quality of life Inventory-Disability (QI-Disability) measure. Qual Life Res 2019;28:783-94.

44 WHO. International classification of functioning, disability and health: ICF. Geneva: World Health Organisation, 2001.

45 Palisano RJ, Chiarello LA, King GA, et al. Participation-based therapy for children with physical disabilities. Disabil Rehabil 2012;34:1041-52.

46 King GA, McDougall J, Palisano R. Goal attainment scaling: its use in evaluating pediatric therapy programs. Physic Occup Therap Pediatr 1999;19:31-52.

47 Dusseldorp E, van Genugten L, van Buuren S, et al. Combinations of techniques that effectively change health behavior: evidence from Meta-CART analysis. Health Psychol 2014;33:1530-40.

48 Downs J, Bebbington A, Woodhead H, et al. Early determinants of fractures in Rett syndrome. Pediatrics 2008;121:540-6.

49 Coster W, Bedell G, Law M, et al. Psychometric evaluation of the participation and environment measure for children and youth. Dev Med Child Neurol 2011;53:1030-7.

50 Romano $\mathrm{A}$, Caprì $\mathrm{T}$, Semino $\mathrm{M}$, et al. Gross motor, physical activity and musculoskeletal disorder evaluation tools for Rett syndrome: a systematic review. Dev Neurorehabil 2020;23:485-501.

51 Stahlhut M, Hill K, Bisgaard A-M, et al. Measurement of Sedentary Behaviors or 'Downtime' in Rett Syndrome. J Child Neurol 2017;32:1009-13.

52 Downs J, Leonard $\mathrm{H}$, Jacoby $\mathrm{P}$, et al. Rett syndrome: establishing a novel outcome measure for walking activity in an era of clinical trials for rare disorders. Disabil Rehabil 2015;37:1992-6.

53 Bruni O, Ottaviano S, Guidetti V, et al. The sleep disturbance scale for children (SDSC). Construction and validation of an instrument to evaluate sleep disturbances in childhood and adolescence. J Sleep Res 1996;5:251-61.

54 Mount RH, Charman T, Hastings RP, et al. The Rett syndrome behaviour questionnaire (RSBQ): Refining the behavioural phenotype of Rett syndrome. J Child Psychol Psychiatry 2002;43:1099-110.

55 Anderson A, Wong K, Jacoby P, et al. Twenty years of surveillance in Rett syndrome: what does this tell us? Orphanet $J$ Rare Dis 2014;9:87.

56 Cass H, Reilly S, Owen L, et al. Findings from a multidisciplinary clinical case series of females with Rett syndrome. Dev Med Child Neurol 2003;45:325-37.

57 Dyke P, Bourke J, Llewellyn G, et al. The experiences of mothers of young adults with an intellectual disability transitioning from secondary school to adult life. J Intellect Dev Disabil 2013;38:149-62.

58 Coster W, Law M, Bedell G, et al. Development of the participation and environment measure for children and youth: conceptual basis. Disabil Rehabil 2012;34:238-46. 